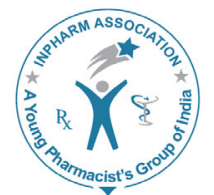

\title{
Formulation and characterization of anti-alzheimer's drug loaded chitosan nanoparticles and its In vitro biological evaluation
}

\author{
Natarajan Tamilselvan, Chellan Vijaya Raghavan* \\ Department of Pharmaceutics, PSG College of Pharmacy, Peelamedu, Coimbatore-641004, INDIA
}

\begin{abstract}
Objective: The main objective of the study is to formulate hydrophilic drug loaded sustained release nanoparticles with the size of $200 \mathrm{~nm}$ and to increase the encapsulation efficiency of the drug. Methodology: The nanoparticles were prepared by simple ionic gelation method using various concentrations of chitosan and TPP. The prepared nanoparticles were evaluated for particle size, shape, charge, encapsulation efficiency, in vitro drug release and in vitro cytotoxicity. Results: The optimized drug loaded nanoparticles showed the size of $125 \pm 4 \mathrm{mn}$ with PDI $0.25 \pm 0.05$, potential of $+40 \pm 2 \mathrm{mV}$, encapsulation efficiency of $65.5 \pm 1.2 \%$ and the drug release of $68.4 \pm 1.6 \%$ with an initial burst effect up to one hour followed by sustained release up to $24 \mathrm{hrs}$. Further the optimized formulation was subjected to investigate the cytotoxicity of CS-NP in SH-SY-5Y cell lines it revealed that the cell viability was above $90 \%$ without any toxicity. Conclusion: These preliminary results demonstrate that the possibility of delivering hydrophilic drugs to brain with enhanced encapsulation efficiency.
\end{abstract}

Key words: Chitosan, Hydrophilic drugs, Nanoparticles, Cytotoxicity, Blood brain barrier.

\section{INTRODUCTION}

The neurodegenerative disorders (NDs) include a variety of conditions that gradually impairs patient memory and cognition in the geriatric population. Alzheimer's disease (AD) affects 24.3 million people worldwide and considered as one of the most severe progressive socio-economical and

\begin{tabular}{|c|c|}
\hline \multicolumn{2}{|c|}{ Access this article online } \\
\hline Journal Sponsor & \multirow[b]{2}{*}{$\begin{array}{l}\text { Website: } \\
\text { www.jyoungpharm.org }\end{array}$} \\
\hline \multirow{2}{*}{ www.phcog net } & \\
\hline & $\begin{array}{l}\text { DOI: } \\
\text { 10.5530/jyp.2015.1.6 }\end{array}$ \\
\hline
\end{tabular}

medical burden all over the world. ${ }^{1}$ Alzheimer's disease is the most common ND s in this century. The etiology of the disease is unclear and some of the factors that are thought to play a crucial role in its pathogenesis include oxidative stress, abnormal proteins and excessive accumulation and reduced acetylcholine levels. ${ }^{2}$ One of the major factor that hinder the drug discovery and development of effective delivery system for the treatment and prevention of $\mathrm{AD}$ due to presence of $\mathrm{BBB}$ which hamper the delivery of anti Alzheimer's drug to brain. ${ }^{3}$ The diffusion of drugs from blood across BBB depends on the physicochemical properties such as lipid solubility, low molecular weight and positive charge. To overcome this difficulty several strategies have been developed to enhance the availability

\footnotetext{
*Address for correspondence:

Dr. Chellan Vijaya Raghavan, Professor, Department of Pharmaceutics, PSG College of Pharmacy, Peelamedu, Coimbatore-641004 Email:drvijayaragha@gmail.com
} 
drugs in the brain.

In recent years the interest in developing drug delivery systems to target brain has lead to the development of various colloidal carriers are employed like liposomes, ${ }^{4}$ polymeric nanoparticle ${ }^{5}$ solid lipid nanoparticle ${ }^{6}$ and dendrimers. ${ }^{7}$ Among the various nanocarriers used polymeric NPs are promising carrier because of its ability to open tight junctions ( $\mathrm{Tj}$ ) of $\mathrm{BBB}$, They effectively mask the membrane barrier limiting the characterization of drug molecule thus prolonging drug release and protecting against enzymatic degradation. Nanoparticles made up of hydrophilic polymers like chitosan have better advantages in terms of like prolonged circulation and nanoparticles with particle size less than $200 \mathrm{~nm}$ avoids opsonisation. ${ }^{8}$

Chitosan is a natural hydrophilic polysaccharide copolymer of glucosamine and $\mathrm{N}$-acetylglycosamine. It is considered as a safe excipient due to its biocompatibility, biodegradability and lack of toxicity. ${ }^{9}$ Further it is cationic in nature and posses mucoadhesive property it will enhance the cellular uptake by ionic interaction. Recently it has received lots of interest for drug delivery as well as biomedical applications like wound healing ointment and dressings, ${ }^{10}$ artificial membranes ${ }^{11}$ contact lenses and bandages. ${ }^{12}$

Tacrine 9-amino-1, 2, 3, 4-tetrahydroacridine was the first acetylcholinesterase inhibitor licensed for the treatment of Alzheimer disease. ${ }^{13}$ Tacrine is readily absorbed and has absolute bioavailability is about $17 \% \pm 13 \%$ which is attributed to very high first-pass metabolism and multiple dose leads to cardiotoxicty .Hence alternative delivery system have been developed to decrease the first pass metabolism and avoiding multiple dosing system. However because of its poor penetration across BBB, it is often necessary to take higher doses of tacrine resulting in cholinergic side effects

The present study was aimed at formulation and characterization of sustained release nanoparticulate system for tacrine with particle $200 \mathrm{~nm}$. Additionally the nanoparticles have been evaluated for the cytotoxicity in SH-SY-5Y human neuroblastoma cell lines

\section{MATERIALS AND METHODS}

\section{Materials and Methods}

Tacrine hydrochloride and chitosan (Medium Mol.Wt, Viscosity of $200 \mathrm{cps}$ ) were purchased from Sigma Aldrich, U.S.A, Sodium tri poly phosphate was purchased from Loba Cheme, glacial acetic acid was obtained from Fishier
Scientific, Dialysis membrane with a molecular weight cut off of 12,000-14,000 daltons was purchased from HiMedia laboratories, Mumbai, Potassium dihydrogen phosphate, tetra butyl methyl ether, ortho phosphoric acid, sodium hydroxide, were of analytical grade and acetonitrile used was HPLC grade

\section{Preparation of chitosan nanoparticles}

Tacrine loaded CS-NPs were prepared using an ionic gelation method. ${ }^{14}$ Determinate weights of chitosan were dissolved in glacial acetic acid 1\% (v/v) $10 \mathrm{mg}$ of tacrine was added to the above solution under constant magnetic stirring, followed by addition of aqueous TPP solution in a drop wise manner. Then the solution was kept on constant magnetic stirring for $30 \mathrm{~min}$ and sonicated using probe sonicator (Vibra Sonics). The nanoparticle suspension was centrifuged at $13,000 \mathrm{rpm}$ and $4^{\circ} \mathrm{C}$ for 30 min using Eppendrof Ultracentrifuge to remove excessive amounts of TPP and unencapsulated tacrine. The pellets were dispersed in deionised water. Finally, NPs were lyophilized for $24 \mathrm{~h}$ using freeze dryer (Lyodel) for storage in powdered form.

\section{Physiochemical characterization of nanoparticles}

\section{Particlesizeand Zeta potential using photon correlation spectroscopy}

The average hydrodynamic diameter and polydispersity index (PDI) of the formulated nanoparticles were determined by dynamic light scattering (DLS) analysis using Zetasizer Nano ZS90 (Malvern Instruments limited, UK), $1 \mathrm{ml}$ of sample of nanoparticles dispersion was placed in disposable cuvettes for particle size measurements. Each experiment was conducted in triplicate. The electrophoretic mobility (zeta potential) measurements were made using the Malvern Zetasizer (Nano ZS90, Malvern Instruments) at $25^{\circ} \mathrm{C}$. Samples were diluted with double distilled water. ${ }^{15}$

\section{Transmission electron microscopy (HRTEM)}

The surface morphology of the prepared NPs was determined for by using transmission electron microscopy (HRTEM). A drop of nanosuspension was placed on a carbon film coated copper grid for TEM. Studies were performed at $80 \mathrm{kv}$ using JOEL JEM 2100, Japan equipped with selected area electron diffraction pattern (SAED). The copper grip was fixed in to sample holder and placed in a vacuum chamber of the transmission electron microscope and observed under low vacuum and TEM images were recorded.

\section{Atomic Force Microscopy (AFM)}


The surface properties of drug loaded nanoparticles were visualized by an atomic force microscope (Nova NTEGRA prima, Russia) Russia) under normal atmospheric conditions. Explorer atomic force microscope was in tapping mode, using high-resonant-frequency ( $\mathrm{F} 0=4-150$ $\mathrm{kHz}$ ) pyramidal cantilevers with silicon probes having force constants of $0.35-6.06 \mathrm{~N} / \mathrm{m}$. Scan speeds were set at 2 $\mathrm{Hz}$. The samples were diluted 10 times with distilled water and then dropped onto glass slides, followed by vacuum drying during 24 hours at $25^{\circ} \mathrm{C}$. Height measurements were obtained using AFM image analysis software (Multimode Scanning probe microscope (NTMDT, NTEGRA prima, Russia)

\section{Encapsulation efficiency}

Nanoparticles were separated from aqueous phase by ultracentrifugation (Eppendrof) at $13000 \mathrm{rpm}$ and $4^{\circ} \mathrm{C}$ for 45 minutes. The supernatants were collected and evaluated for tacrine residue by UV. The encapsulation efficiency (EE) was determined indirectly by measurement of the amount of free tacrine in the supernatant after ultracentrifugation and was calculated according to the following equation:

$\mathrm{EE}=\frac{\text { Amount of total drug }- \text { Amount of free drug in supernant }}{\text { Amount of total drug }} \times 100$

\section{In vitro release}

A modified dialysis method was used to evaluate the in vitro release of tacrine-loaded chitosan NPs. Two millilitres of nanoparticles suspension (corresponding to $2 \mathrm{mg}$ of tacrine) was placed in a dialysis bag (cellophane membrane, molecular weight cut off 10,000-12,000, Hi-Media, India), which was tied and placed into $20 \mathrm{ml}$ of phosphate buffer (0.1 M, pH 7.4) maintained at $37^{\circ} \mathrm{C}$ with continuous magnetic stirring. At selected time intervals, aliquots were withdrawn from the release medium and replaced with the same amount of phosphate buffer. The sample was assayed spectrophotometrically for tacrine at $335 \mathrm{~nm} .{ }^{16}$

\section{In vitro release kinetics}

The drug release data were computed using DDsolver, which is an Excel plugin module and the resultant data were fitted to the Korsmeyer-Peppas exponential equation (1) to establish the mechanism of drug release where $Q$ is the percentage of drug released at time $t$ and $k$ is a constant incorporating the structural and geometric characteristics of the dosage form. The diffusional exponent $n$ is an important indicator of the mechanism of drug transport from the dosage form. ${ }^{17}$

$$
Q=k t^{n}
$$

\section{In vitro cytotoxicity of nanoparticles}

Human SH-SY5Y cells were obtained from National Center for Cell Science (NCCS), Pune. Cells were cultured in MEM supplemented with non-essential amino acid, ham F-12, 10\% fetal bovine serum, $2 \mathrm{mmol} / \mathrm{L}$ L-glutamine, penicillin $(100 \mathrm{U} / \mathrm{mL})$ and streptomycin $(100 \mu \mathrm{g} / \mathrm{mL})$, and maintained at $37^{\circ} \mathrm{C}$ with $5 \% \mathrm{CO}_{2}$ in a humid environment. The medium was replaced twice every week and the cellular viability was assessed using MTT assay. SH-SY5Y cells were seeded at a density of $1 \times 10^{4}$ cells per well in 96-well plates for $24 \mathrm{~h}$ in $10 \%$ serum condition. Gradual reduction of serum $(5 \%)$ was done in consecutive day and finally cells were maintained serum free for 16 hrs. wells were divided in triplicate as normal control group, placebo and 2 different formulations of tacrine solution and nanoparticle at the dose of $10 \mu \mathrm{g}$ and $100 \mu \mathrm{g}$. Drug formulations were incubated with cells for 24 hrs. MTT at a concentration of $5 \mathrm{mg} / \mathrm{ml}$ was prepared and $20 \mu \mathrm{l}$ of MTT solution was added to each well and incubated for $4 \mathrm{hrs}$. Followed by MTT incubation, medium containing MTT was discarded and $50 \mu \mathrm{l}$ of DMSO was added to each well to dissolve formazan crystals. Optical density was measured at $570 \mathrm{~nm}$. Percentage toxicity was measured against control.

\section{RESULT AND DISCUSSION}

In the present study we developed a nanoparticulate system which is composed of hydrophilic polymer chitosan possessing the following advantage like 1) obtaining NP spontaneously by mild agitations absence of organic solvents and high temperature 2) obtaining NP with positive charge which could enhance the cellular uptake chitosan produces low to high positive charge which could enhance the cellular uptake and has mucoadhesive property.

\section{Conditions for formation tacrine loaded chitosan nanoparticles}

Chitosan NPs were prepared by simple scale up ionotropic gelation method similar to the method developed. ${ }^{18}$ Chitosan is a cationic polyelectrolyte the nanoparticles were formed by inducing the gelation by controlling its interaction with polyanion TPP which leads to reduce the aqueous solubility of CS this system based on inter and intramolecular linkages created between TPP and positive charge of charged amino groups of CS which are responsible for the successful formation of the 
Table 1: Optimization of nanoparticles (CS-NP) on the basis of SC/TPP ratio

\begin{tabular}{llllllll}
$\begin{array}{l}\text { Formulation } \\
\text { code }\end{array}$ & CS (\%) & TPP $(\%)$ & Size $(\mathbf{n m})$ & PDI & $\begin{array}{l}\text { Zeta potential } \\
(\mathbf{m V})\end{array}$ & EE $(\%)$ & Physical appearance and opacity \\
\hline CT1 & 0.2 & 0.2 & $414 \pm 4$ & $0.49 \pm 0.03$ & $+6 \pm 1$ & $46.9 \pm 1.8$ & opalescent suspension \\
CT2 & 0.4 & 0.2 & $290 \pm 6$ & $0.31 \pm 0.31$ & $+12 \pm 1.2$ & $55.5 \pm 1.1$ & opalescent suspension \\
CT3 & 0.6 & 0.2 & $125 \pm 4$ & $0.25 \pm 0.05$ & $+40 \pm 2$ & $65.5 \pm 1.2$ & opalescent suspension \\
CT4 & 0.2 & 0.4 & $155 \pm 10$ & $0.34 \pm 0.07$ & $+31 \pm 2.5$ & $70.6 \pm 1.5$ & opalescent suspension \\
CT5 & 0.4 & 0.4 & $1574 \pm 2$ & $0.31 \pm 0.04$ & $+5.7 \pm 1.5$ & $52.6 \pm 2.05$ & Highly opalescent suspension \\
CT6 & 0.6 & 0.4 & $1953 \pm 3$ & $0.28 \pm 0.07$ & $+4.6 \pm 2$ & $42.3 \pm 1.6$ & Highly opalescent Suspension \\
CT7 & 0.2 & 0.6 & $1731 \pm 2$ & $0.42 \pm 0.05$ & $+5 \pm 1.6$ & $47.8 \pm 1.4$ & Highly opalescent suspension \\
CT8 & 0.4 & 0.6 & $2852 \pm 8$ & $0.56 \pm 0.07$ & $+4 \pm 2.5$ & $55.06 \pm 1.9$ & Highly opalescent suspension \\
CT9 & 0.6 & 0.6 & $2764 \pm 6$ & $0.84 \pm 0.08$ & $+3 \pm 3$ & $43.2 \pm 1.7$ & Highly opalescent suspension
\end{tabular}

Optimization of nanoparticles (CS-NP) on the basis of CS/TPP ratio

nanoparticles. The CS/TPP ratio is rate limiting step and controls the size and size distribution of nanoparticles. ${ }^{19}$ In order to obtain nanoparticles under $200 \mathrm{~nm}$ we studied the effect of the CS/TPP ratio on the formation of nanoparticles. The maximum concentration of CS used was up to $6 \mathrm{mg} / \mathrm{ml}$ while the maximum concentration of TPP was $4 \mathrm{mg} / \mathrm{ml}$. The particle size, PDI, drug encapsulation and zeta potential were analyzed and the result are presented in Table: 1 Our results indicated that particle size depend on both CS and TPP concentration that the specific concentration of CS/TPP can only form the nanoparticles with smaller size.

\section{Effect of chitosan concentration}

The role of chitosan concentration $(0.2,0.4 \& 0.6 \%)$ on formation of nanoparticles and its influence on particle size was evaluated. When the amount of TPP was kept constant as $0.2 \%$ and an increase in CS concentration from $0.2 \%$ to $0.6 \%$ showed a decrease in the particle size with favourable
PDI value. When the amount of chitosan exceeded $0.6 \%$ of CS a highly opalescent suspension is formed and it also leads to aggregation. Recent studies reported ${ }^{20}$ that when the concentration of CS is low $(0.6 \%)$ it forms a low viscosity gelation medium resulting in a decrease in liquid phase dispersion, thus promoting formation of smaller particles.

\section{Effect of TPP concentration}

The role of TPP $(0.2,0.4 \& 0.6 \%)$ concentration on particle size formation was studied. The increase in TPP concentration showed an increase in particle size. The TPP concentration with 0.2 and $0.4 \%$ with 0.6 and $0.2 \%$ chitosan forms particle $200 \mathrm{~nm}$ at the same time TPP concentration at 0.4 and $0.6 \%$ with 0.4 and $0.6 \%$ of CS concentration it showed a huge increase in particle size results in microparticles. When TPP concentration above $0.4 \%$ it results in highly opalescent suspension on storage it starts settling of particles.

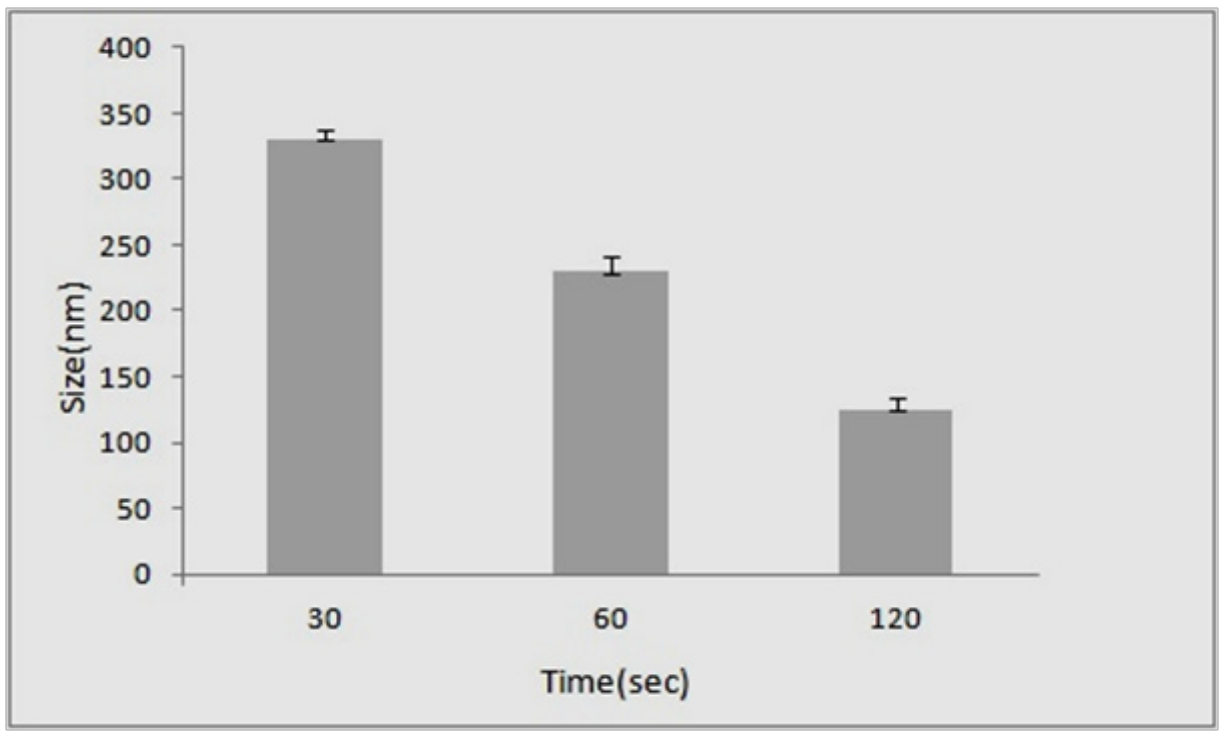

Figure 1: Influence of sonication time on particle size 


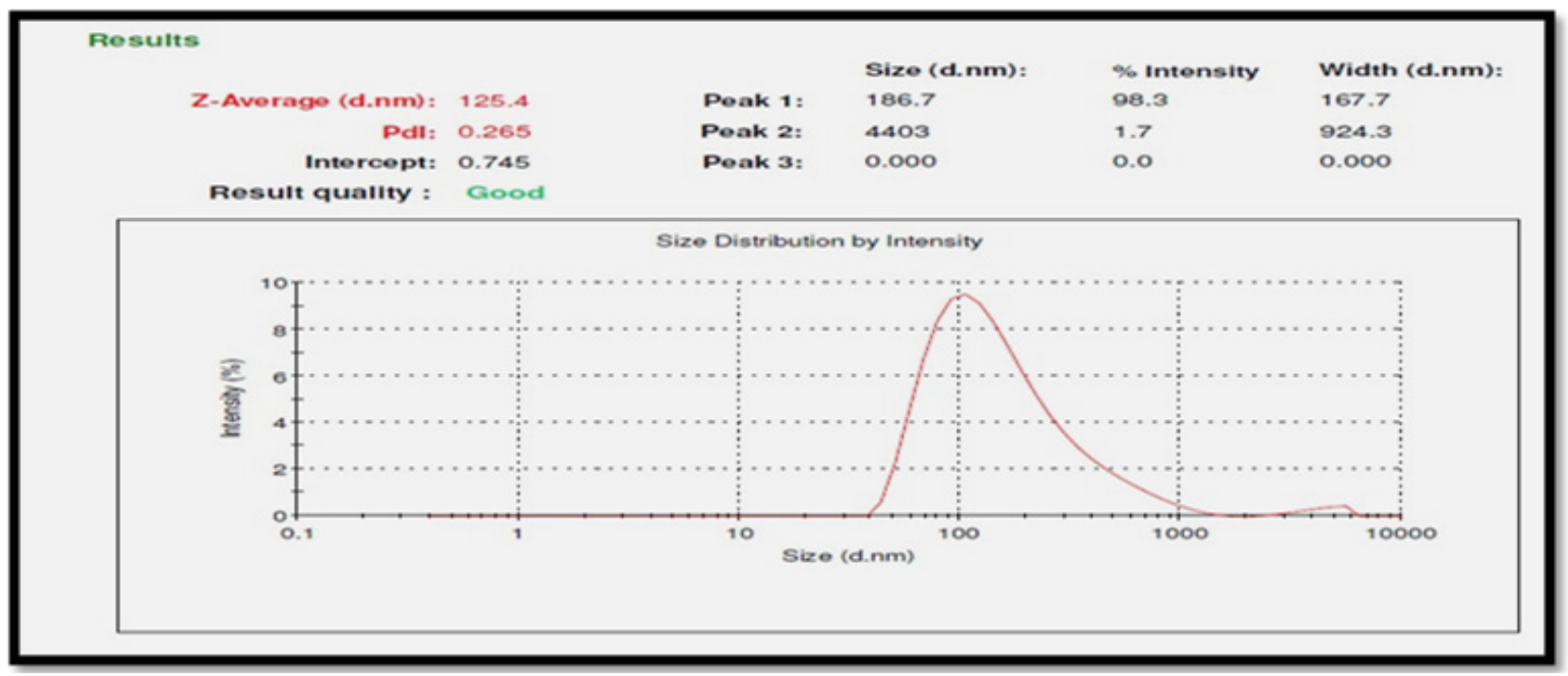

Figure 2: Particle size distibution of tacrine loaded CS-NP containing CS of $0.6 \%$ and TPP $0.2 \%$

\section{Effect of sonication on particle size}

The sonication time in the formation of CS-NP played a crucial role in the formation of smaller size nanoparticles. The smallest nanoparticles $(125 \pm 4 \mathrm{~nm})$ were obtained with the sonication time of two minutes. While employing ultrasonication formation of acoustic cavitations is the main cause for decreasing particle size. Acoustic cavitations by creating a large shear force on the chitosan molecules breaks the particles in to smaller ones. The increase in the sonication time from 30, 60 and 120 seconds showed the decreased particle size presented in (Figure 1). The sonication time beyond two minutes showed no further decrease in particle size

\section{Particle size and Zeta potential}

The nine formulations were prepared with various concentrations of chitosan and TPP. The particle size distribution of prepared CS nanoparticles was ranged from $125 \pm 4$ to $2852 \pm 8 \mathrm{~nm}$. With increasing the concentration of CS we observed decrease in particle size and increase in zeta value. At $0.2 \%$ concentration of TPP the cross linking with chitosan is high $(0.6 \%)$ this result in more compact particle structure and the neutralization degree of charged amino acid is improved leading the good net charge of the particles. Due to the compact structure and net charge the particles prepared at this concentration have a smaller size.

The zeta potential of the prepared CS nanoparticles was ranged from +5 to $+40 \mathrm{mV}$. When increase in the concentration of CS the zeta value increases due to the higher degree of protonation of amino group in the CS molecule with the strong positive charge which leads to the higher zeta potential.

The optimum concentration of CS/TPP was identified as $0.6 \%$ of CS with $0.2 \%$ TPP (CT3) with size of (125 $\pm 4) \mathrm{nm}$ showed in (Figure: 2). the zeta potential for the prepared tacrine loaded CS-NP (CT3) was $40 \pm 2 \mathrm{mV}$ which indicates the good colloidal stability of the prepared CS NP. The TEM images of the prepared tacrine loaded CS-NP (CT3) indicate that nanoparticles were roughly spherical in shape with size of $50 \mathrm{~nm}$ shown in Figure: 3. Further the morphology of the nanoparticles was also analysed using AFM. The 2D and 3D images in (Figure: $4 \mathrm{~A}$ and $4 \mathrm{~B}$ ) indicates that the particles are in sub spherical

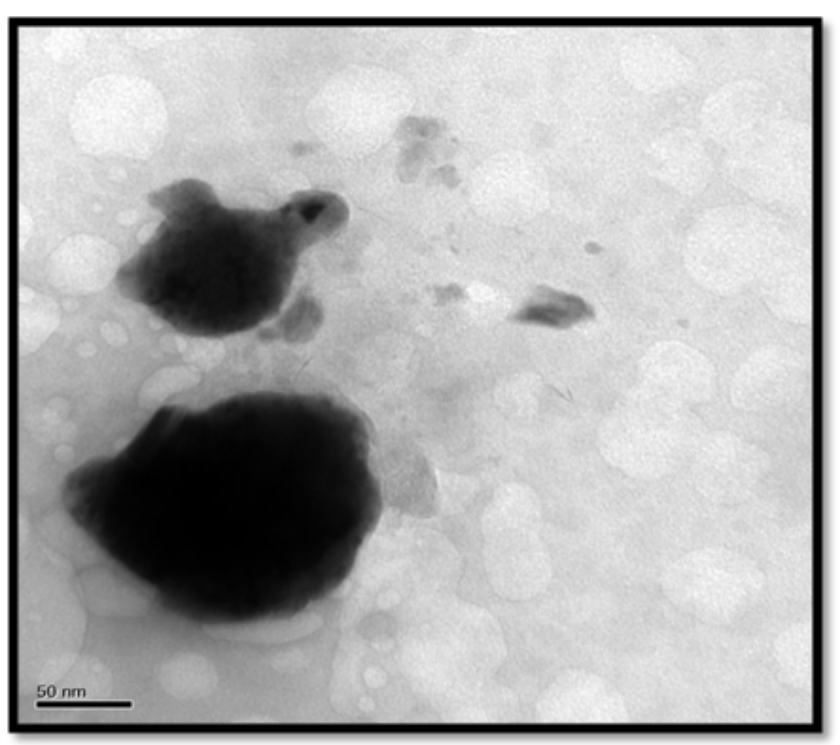

Figure 3: TEM images of tacrine loaded CS-NP

Journal of Young Pharmacists Vol 7 • Issue 1 • Jan-Mar 2015 
shape dense particles with size of $50 \mathrm{~nm}$. The physical state of tacrine loaded CS-NP (CT3) was analysed using selected area electron diffraction pattern (SAED) (Figure 5) showed that entrapped drug was in amorphous or in molecular dispersed state.

The encapsulation efficiency of tacrine loaded CS-NP were ranged from $42.3 \pm 1.6$ to $70.6 \pm 1.5 \%$.The increase in chitosan concentration from 0.2 to $0.6 \%$ increases in encapsulation was observed at constant TPP concentration of $0.2 \%$. Out of these formulations CT3 was selected as the best formulation based on particle size, zeta potential $(>+30 \mathrm{mV})$ and encapsulation of $65.5 \pm 1.2 \%$. The optimized formulation was selected for further studies.

\section{In vitro release study}

The cumulative percentage release of optimized tacrine loaded CS-NP (CT3) and free drug solution (T-sol) was studied in phosphate buffer $\mathrm{pH} 7.4$ and showed in (Figure $6)$. The percentage release was found to be $68.4 \pm 1.6 \%$ up to $24 \mathrm{hrs}$ and percentage release of drug solution was about $96.4 \%$ in $4 \mathrm{hrs}$. The release profile of tacrine loaded CS-NP exhibits a initial release burst release of $20 \%$ in one hour followed by the sustained release of 38\% for following 24 hrs. The observed burst effect was due to the dissociation of drug molecules that were loosely bound to the surface of the chitosan nanoparticles. the second part of the release
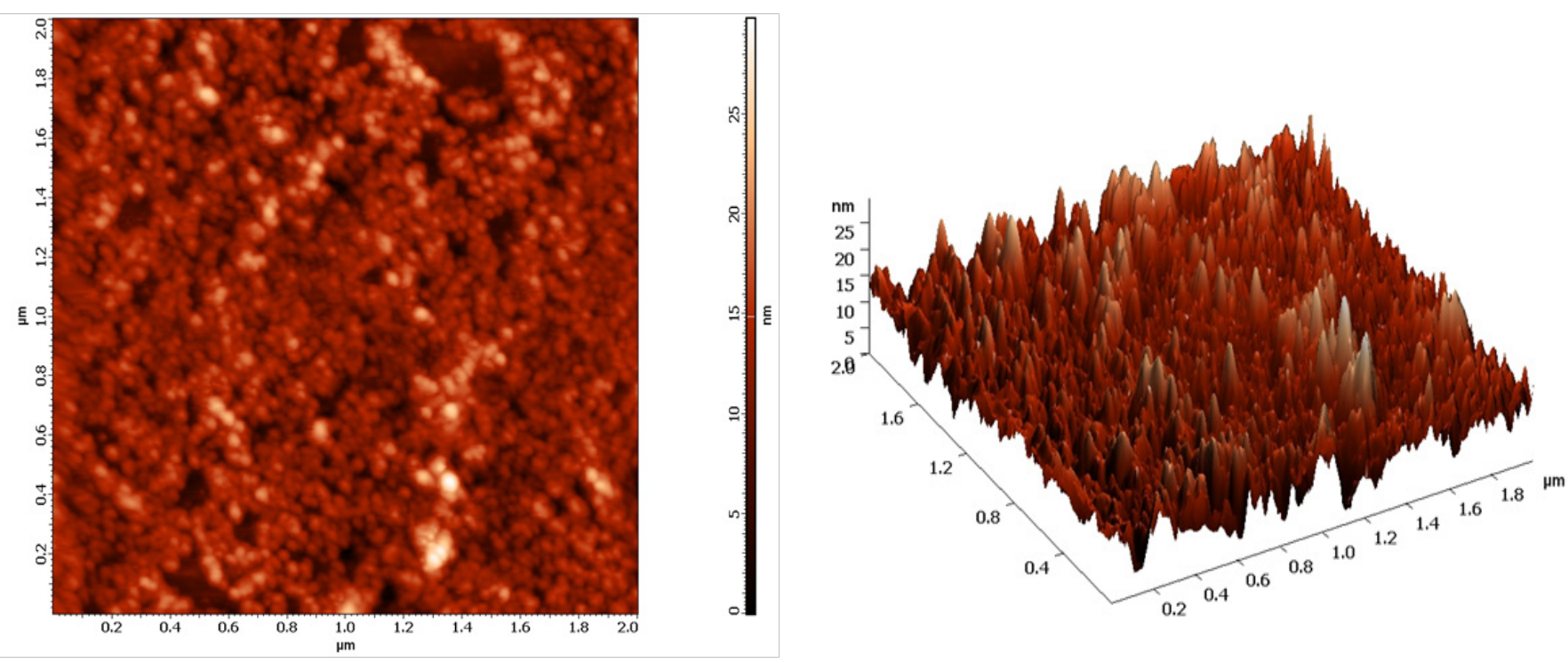

Figure 4: Selected area electron diffraction pattern image of tacrine loaded CS-NP

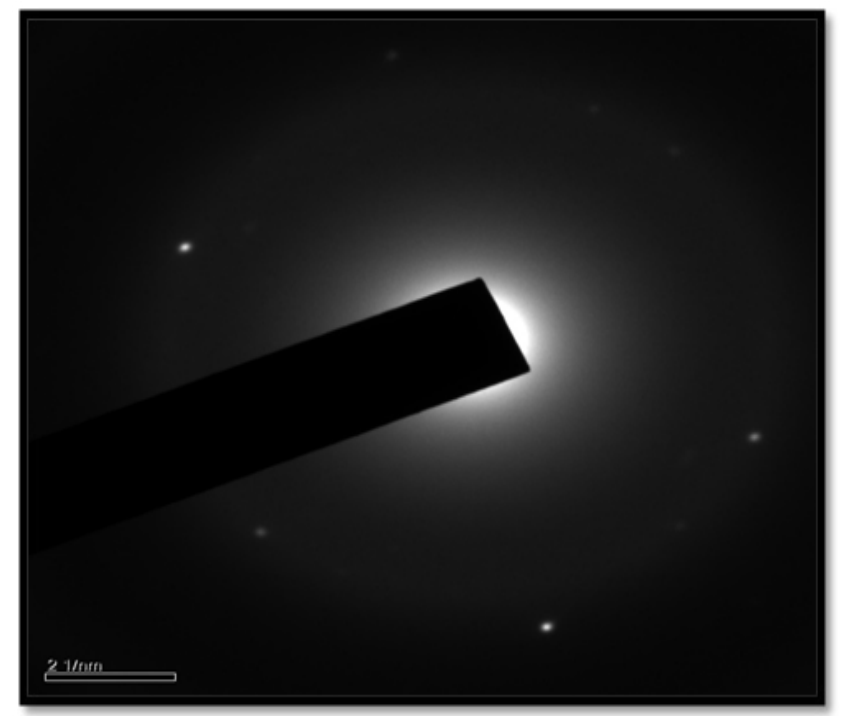

Figure 5: AFM images of tacrine loaded CS-NP (A) 2D (B) 3D images

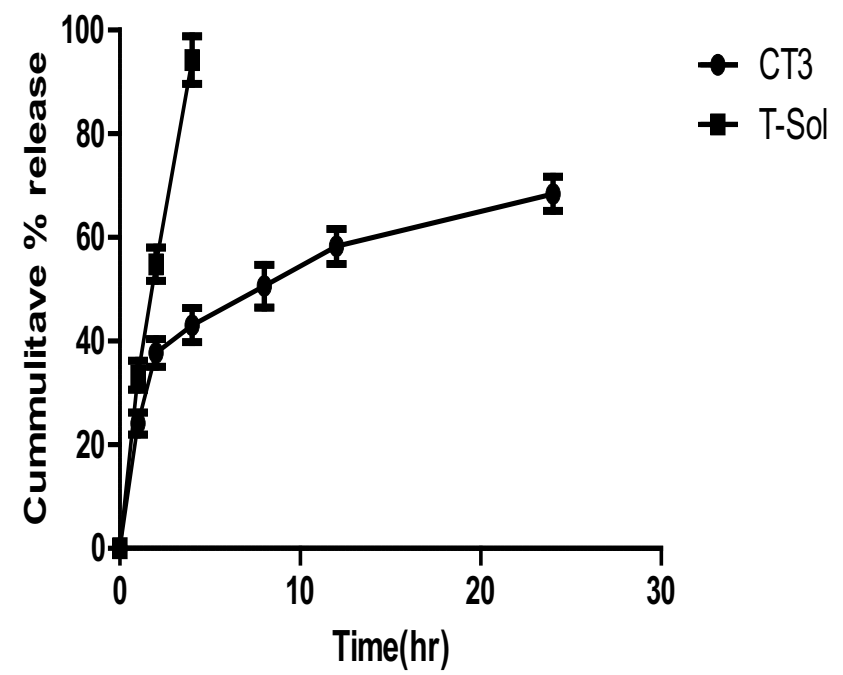

Figure 6: In vitro drug release of tacrine loaded CS-NP 

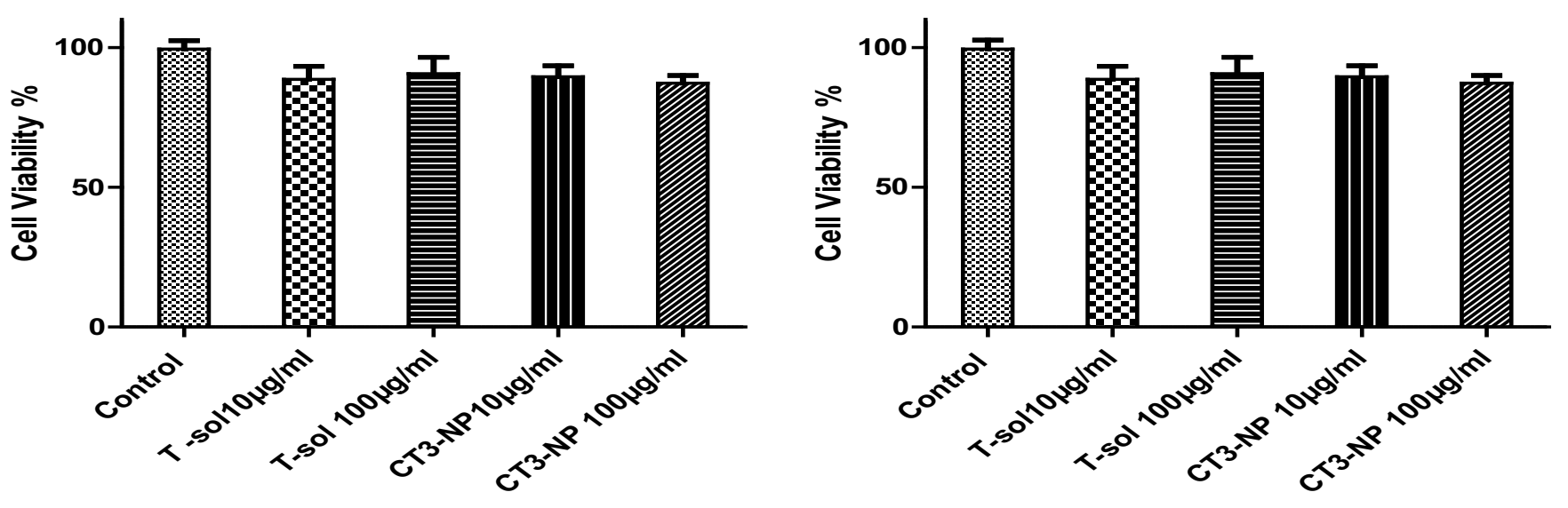

Figure 7: Cell viability of SH-SY-5Y cell line after incubation with T-sol (10 and $100 \mu \mathrm{g} / \mathrm{ml})$ and T-NP (10 and $100 \mu \mathrm{g} / \mathrm{ml})$ at 7A) $12 \mathrm{hr}$ and 7B) $24 \mathrm{hr}$

was slow and sustained release of encapsulated tacrine at an approximately constant rate from the nanoparticles.

In order to investigate the mode of drug release from chitosan nanoparticles. The release data of the optimized formulation were fitted to korsmeyer peppas model it sowed the regression coefficient $\left(\mathrm{r}^{2}\right.$ value) of 0.9916 . And the ' $\mathrm{n}$ ' were in the range of 0.307 it indicates the drug release follows fickian diffusion controlled release pattern.

\section{In vitro cytotoxicity study}

Cytotoxicity of unloaded and tacrine loaded chitosan nanoparticles was evaluated by MTT assay on SH-SY-5Y human neuroblastoma cell lines, it is used extensively to screen novel compounds for neurotoxicity properties. The cells were incubated with pure drug solution and tacrine loaded chitosan nanoparticle at the concentration of 10 and $100 \mu \mathrm{g} / \mathrm{ml}$ for 12 and $24 \mathrm{hrs}$. The results of cell viability $(\%)$ were presented in Figure 7 and 7A. The data suggested that the tacrine loaded nanoparticles at 10 and $100 \mu \mathrm{g} / \mathrm{ml}$ concentration does not produce any toxicity on this cell even at the higher concentration and the cell viability of the formulation was above $90 \%$ this indicates the safety of the tacrine loaded nanoparticles for further use in in vivo.

\section{CONCLUSION}

This study demonstrates the ionic gelation method can be used to load hydrophilic drugs and produce the size of less than $200 \mathrm{~nm}$. The concentration of CS, TPP and sonication time strongly effect the particle size formation of the CS-NP. The CS-NP composed of $0.6 \%$ CS and $0.2 \% \mathrm{TPP}$ was selected as the optimized formulation which produced smaller particle with better encapsulation. In vitro cytotoxicity study suggested the safety of the prepared CS-
NP which can be potential carrier to deliver hydrophilic drugs to target brain. Further In vivo will confirm the targeting efficiency of CS-NP across blood brain barrier to treat Alzheimer's disease.

\section{ACKNOWLEDGEMENT}

The authors thank Indian Council of Medical Research (ICMR), New Delhi for providing a Senior Research Fellowship. The authors thank Dr. K. Balakumar and Mr. Siram karthick for the assistance in characterization of nanoparticles. We also thank Mr. Siva selva Kumar, Mr. Hari Prasad for the assistance in HPLC analysis and Mr. Rangith Kumar for the assistance in animal study.

\section{CONFLICT OF INTEREST}

Authors declare no conflict of interest

\section{REFERENCES}

1. Ferri CP, Prince M, Brayne C, Brodaty H, Fratiglioni L, Ganguli M et al.Global prevalence of dementia: a Delphi consensus study. The Lancet. 2006; 366(9503): 2112-7.

2. Zhang HY. One-compound-multipletargets strategy to combat Alzheimer's disease. FEBS Lett. 2005; 579(24):5260-4.

3. Lockman PR, Mumper RJ, Khan MA, Allen DD. Nanoparticle technology for drug delivery across blood-brain barrier. Drug Dev. Ind. Pharm. 2002; 28(1): 1-13.

4. Mori N, Kurokouchi A, Osonoe K, Saitoh H, Ariga K, Suzuki K, et al . Liposome entrapped phenytoin locally suppresses amygdaloidal epileptogenic focus created by db-CAMP/EDTA in rats. Brain Res. 1995; 703(1-2): 184-90.

5. Kreuter J, Ramge P, Petrov V, Hamm S, Gelperina SE, Engelhardt B. Direct evidence that polysorbate-80-coated poly (butylcyanoacrylate) nanoparticles deliver drugs to the CNS via specific mechanisms requiring prior binding of drug to the nanoparticles. Pharm. Res. 2003; 20(3): 409-16.

6. Shuting K, Feng Y, Ying W, Yilin S, Nan Y, Ling Y. The blood-brain barrier penetration and distribution of PEGylated fluorescein-doped magnetic silica nanoparticles in rat brain. Biochem. Biophys. Res. 2010; 394(4): 871-6.

7. Hai H, Yan L, Xin RJ, Ju D, Xue Y, Wan-L L et al. The blood-brain barrier penetration and distribution of PEGylated fluorescein-doped magnetic silica nanoparticles in rat brain. Biomaterials. 2011; 32(4): 478-87.

8. Ganeshchandra S, Keishiro T, Kimiko M. Biodistribution of colloidal gold 
nanoparticles after intravenous administration: Effect of particle size. Colloids and Surfaces B: Biointerfaces. 2008; 66(2): 274-80.

9. Felt $O$, Buri P, Gurny R. Chitosan: a unique polysaccharide for drug delivery. Drug Dev. Ind. Pharm. 1998; 24(11): 979-93.

10. Tachihara K, Onishi H, Machida Y. Preparation of silver sulfadiazine containing spongy membranes on chitosan and chitin chitosan mixture and their evaluation as burn wound dressings. J. Pharmaceutical Science and Technology Japan. 1997; 57(3): 159-67.

11. Amiji MM. Permeability and blood compatibility properties of chitosan poly (ethylene oxide) blend membranes for haemodialysis. Biomaterials.1995; 16(8):593-9.

12. Kaur IP, Smitha R. Penetration enhancers and ocular bioadhesive: two new avenues for ophthalmic drug delivery. Drug Dev Ind Pharm. 2002; 28(4): 353-69.

13. Reichmann WE. Current pharmacologic options for patients with Alzheimer's disease. Ann Gen Hosp Psychiatr. 2003; 2(1): 1

14. Arya G, Vandana M, Acharya S, Sahoo, SK. Enhanced antiproliferative activity of Herceptin (HER2)-conjugated Gemcitabine-loaded chitosan nanoparticle in pancreatic cancer therapy. Nanomed. Nanotechnol. Biol. Med. 2011; 7(6): 859-70.

15. Siram k, Vijaya Raghavan C, Tamilselvan N, Balakumar K, Habibur Rahman SM, Vamshi Krishna K et.al. Solid lipid nanoparticles of diethylcarbamazine citrate for enhanced delivery to the lymphatics: in vitro and in vivo evaluation. Expert Opin. Drug Deliv. 2014; 11(8): 1-15.

16. Hua $Y$, Jianga $X$, Dinga $Y$, Zhanga L, Yanga $C$, Zhang J, Preparation and drug release behaviours of nimodipine-loaded poly (caprolactone)-poly (ethyleneoxide)-polylactide amphiphilic copolymer nanoparticles. Biomaterials 2003; 24(13): 2395-404.

17. Zhang Y, Huo M, Zhou J, Zou A, Li W, Yao C et al. "DD Solver: an add-in program for modelling and comparison of drug dissolution profiles." AAPS Journal. 2010; 12(3): 263-71.

18. Calvo P, Remuñán-López C, Vila-Jato JL, Alonso MJ. Novel Hydrophilic ChitosanPolyethylene Oxide Nanoparticles as Protein Carriers. J. Applied Polymer Sci. 1997; 63(1): 125-32.

19. Emmanuel NK, Sofia AP, Dimitrios NB, George EF. Insight on the Formation of Chitosan Nanoparticles through Ionotropic Gelation with Tripolyphosphate. Molecular Pharmaceutics. 2012; 9(10): 2856-62.

20. Mohammadpour D N , Eskandari R, Avadi MR, Zolfagharian H, Mir Mohammad SA, Rezayat M. Preparation and in vitro characterization of chitosan nanoparticles containing Mesobuthus eupeus scorpion venom as an antigen delivery system. The Journal of Venomous Animals and Toxins including Tropical Diseases. 2012 18(1): $44-52$ 